\title{
ON PROPERTIES OF $t$-FUZZY MODULES AND UNIFORM $t$-FUZZY MODULES
}

\author{
Samruam Baupradist ${ }^{1}$ And Burhan Chemat \\ Department of Mathematics and Computer Science \\ Faculty of Science \\ Chulalongkorn University \\ Bangkok 10330, Thailand \\ e-mail: samruam.b@chula.ac.th \\ chengyailek@gmail.com
}

\begin{abstract}
In this paper, we extend the idea of fuzzy modules and uniform fuzzy modules to the concepts of $t$-fuzzy modules and uniform $t$-fuzzy modules, respectively. We give some characterizations and properties of $t$-fuzzy modules and uniform $t$-fuzzy modules.
\end{abstract}

Keywords: uniform fuzzy modules, $t$-fuzzy modules, uniform $t$-fuzzy modules.

2010 Mathematics Subject Classification: 08A72, 03E72.

\section{REFERENCES}

[1] C. Burhan and B. Samruam, On properties of unifrom fuzzy modules and semiuniform fuzzy modules, (will be appear).

[2] P. Dheena and G. Mohanraaj, T-fuzzy ideals in rings, Int. J. Comp. Cogn. 9 (2011) 98-101. doi:10.12732/ijpam.v89i3.5

[3] D. Dubois and H. Prade, Fuzzy Sets and Systems: Theory and Applications (New York, Academic Press, 1980). doi: $10.1057 /$ jors. 1982.38

[4] J.S. Golan, Making modules fuzzy, Fuzzy Sets and Systems 32 (1989) 91-94. doi:10.1016/0165-0114(89)90089-4

\footnotetext{
${ }^{1}$ Corresponding author.
} 
[5] U. Medhi and H.K. Saikia, On T-fuzzy essential ideals of rings, Int. J. Pure Appl. Math. 89 (2013) 343-352.

doi:10.12732/ijpam.v89i3.5

[6] X.V. Nagoita and D.A. Ralescu, Applications of Fuzzy Sets and System Analysis (Basel, Birkhauser, 1975).

doi:10.1109/TSMC.1977.4309811

[7] F. Pan, Fuzzy finitely generated modules, Fuzzy Sets and Systems 21 (1987) 105-113.

doi:10.1016/0165-0114(87)90156-4

[8] F. Pan, Fuzzy quotient modules, Fuzzy Sets and Systems 28 (1988) 85-90.

doi:10.1016/0165-0114(88)90118-2

[9] R. Rasuli, Fuzzy modules over a t-norm, Int. J. Open Prob. Compt. Math. 9 (2016) 12-18. doi:10.12816/0033740

[10] A. Rosenfeld, Fuzzy groups, J. Math. Anal. Appl. 35 (1971) 512-517. doi:10.1016/0022-247x(71)90199-5

[11] M. Ujwal and K. S. Helen, On T-fuzzy essential ideals of rings, Int. J. Pure and Appl. Math. 89 (2013) 345-352. doi:10.12732/ijpam.v89i3.5

[12] L.A. Zadeh, Fuzzy sets, Inform. and Control 8 (1965) 228-258. doi:10.21236/ad0608981

Received 3 June 2019

Revised 1 August 2019 Accepted 1 September 2019 